\title{
Calculation and Properties of Zonal Polynomials
}

\author{
Lin Jiu and Christoph Koutschan
}

\begin{abstract}
We investigate the zonal polynomials, a family of symmetric polynomials that appear in many mathematical contexts, such as multivariate statistics, differential geometry, representation theory, and combinatorics. We present two computer algebra packages, in SageMath and in Mathematica, for their computation. With the help of these software packages, we carry out an experimental mathematics study of some properties of zonal polynomials. Moreover, we derive and prove closed forms for several infinite families of zonal polynomial coefficients.
\end{abstract}

Mathematics Subject Classification (2010). Primary 05E05; Secondary 33C20 33C70 15B52 65 C60.

Keywords. zonal polynomial, symmetric function, integer partition, Laplace-Beltrami operator, Wishart matrix, hypergeometric function of a matrix argument.

\section{Introduction}

At the beginning of our study, we recall the generalized hypergeometric function ${ }_{p} F_{q}$, defined as the infinite series

$$
{ }_{p} F_{q}\left(\begin{array}{c}
a_{1}, \ldots, a_{p} \\
b_{1}, \ldots, b_{q}
\end{array} \mid z\right):=\sum_{n=0}^{\infty} \frac{\left(a_{1}\right)_{n} \cdots\left(a_{p}\right)_{n}}{\left(b_{1}\right)_{n} \cdots\left(b_{q}\right)_{n}} \cdot \frac{z^{n}}{n !},
$$

where for positive integer $m,(a)_{m}:=a(a+1) \cdots(a+m-1)$ is the Pochhammer symbol. What is less well-known is a remarkable generalization of this hypergeometric function of a matrix argument, as follows.

Definition 1.1. Given an $m \times m$ symmetric, positive-definite matrix $Y$, the hypergeometric function ${ }_{p} F_{q}$ of matrix argument $Y$ is defined as

$$
{ }_{p} F_{q}\left(\begin{array}{c}
a_{1}, \ldots, a_{p} \\
b_{1}, \ldots, b_{q}
\end{array} \mid Y\right):=\sum_{n=0}^{\infty} \sum_{\lambda \in \mathcal{P}_{n}} \frac{\left(a_{1}\right)_{\lambda} \cdots\left(a_{p}\right)_{\lambda}}{\left(b_{1}\right)_{\lambda} \cdots\left(b_{q}\right)_{\lambda}} \cdot \frac{\mathcal{C}_{\lambda}(Y)}{n !},
$$

where

- $\mathcal{P}_{n}$ is the set of all integer partitions of $n$, in which, every partition $\lambda \in \mathcal{P}_{n}$ is defined to be a tuple $\lambda=\left(\lambda_{1}, \ldots, \lambda_{k}\right)$ such that $\lambda_{1} \geq \lambda_{2} \geq \cdots \geq \lambda_{k} \geq 1$ and $\lambda_{1}+\cdots+\lambda_{k}=n$;

- $(a)_{\lambda}$ is the generalized Pochhammer symbol, defined as

$$
(a)_{\lambda}=(a)_{\left(\lambda_{1}, \ldots, \lambda_{k}\right)}:=\prod_{i=1}^{k}\left(a-\frac{i-1}{2}\right)_{\lambda_{i}} ;
$$

LJ was supported by the Austrian Science Fund (FWF): P29467-N32.

CK was supported by the Austrian Science Fund (FWF): P29467-N32 and F5011-N15.

This is a post-peer-review, pre-copyedit version of an article published in Mathematics in Computer Science. The final authenticated version is available online at: http://dx.doi.org/10.1007/s11786-020-00458-0 
- and finally $\mathcal{C}_{\lambda}(Y)$ denotes the zonal polynomial of $Y$, indexed by a partition $\lambda$, which is a symmetric homogeneous polynomial of degree $n$ (see Section 2) in the eigenvalues $y_{1}, \ldots, y_{m}$ of $Y$, satisfying

$$
\sum_{\lambda \in \mathcal{P}_{n}} \mathcal{C}_{\lambda}(Y)=(\operatorname{tr} Y)^{n}=\left(y_{1}+\cdots+y_{m}\right)^{n} .
$$

By noting that the zonal polynomial is zero whenever $k$, the number of parts of $\lambda$, exceeds the dimension of $Y$ (see Remark 3.4), one recognizes that (1.2) indeed specializes to (1.1) when $Y$ is a $(1 \times 1)$-matrix, since for each $n$, only the partition $\lambda=(n)$ contributes.

The hypergeometric function of a matrix argument is used in multivariate statistics, in connection with the Wishart distribution [21]; see Section 2.1] and [3] for an introduction. For example, the extreme eigenvalues of random matrices can be expressed in terms of this hypergeometric function [2, 8]. However, the numerical evaluation of ${ }_{p} F_{q}$ functions of a matrix argument is a notorious problem in multivariate distribution theory [10].

Recent progress on the numerical evaluation is based on the holonomic gradient method [16]. In the case $p=q=1$, it is known that the hypergeometric function ${ }_{1} F_{1}(a ; c ; Y)$ satisfies a holonomic system of partial differential equations [14] in the variables $y_{1}, \ldots, y_{m}$, whose holonomic rank is $2^{m}$. Hashiguchi et al. [4] use it to study the cumulative distribution of the largest eigenvalue of a Wishart matrix. The problem of specializing this high-dimensional holonomic system to singular regions has been addressed in [17]. As an application, the Wishart distribution arises in the performance analysis of wireless communication systems under Rayleigh fading [18]. For evaluating the hypergeometric function of a matrix argument using the holonomic gradient method, one needs to know the first few zonal polynomials in order to get accurate initial conditions.

A comprehensive introduction to zonal polynomials [1, §35.4] was given by Takemura [20]. Interestingly, these polynomials also appear in completely different mathematical contexts. No direct formula for their calculation is known, but only partial results [12, 15]. There exist software packages in Maple [19] and SageMath ${ }^{1}$ to compute with them.

In Section 2 we give a survey of different definitions of zonal polynomials. In Section 3 we recall a recursive method by Muirhead [15] to calculate zonal polynomials. Note that there is no general direct formula to obtain the coefficients of the zonal polynomial. In the following we will give such formulas for some special families of zonal polynomial coefficients: In Section 4 we present conditions under which a coefficient vanishes, and in Section 5 we derive closed forms for the coefficients at the two extremal corners of the coefficient matrix. Then we give a complete closed form for zonal polynomials in two variables (Section 6) and some partial results for three and four variables (Section 77). Finally, we explain some details of our software packages, see Sections 8 and 9 that were written in the frame of the current work.

\section{Definitions of zonal polynomials}

We shall summarize four different definitions of zonal polynomials involving statistics, differential geometry, representation theory and combinatorics. Namely, each subsection will present one aspect. First of all, we need an important linear space.

Definition 2.1. Let $V_{n}$ be the space of symmetric homogeneous polynomials of degree $n$ in the variables $y_{1}, \ldots, y_{m}$, including the zero polynomial. Namely, if $f \in V_{n}$, we have

- $\operatorname{deg} f=n$ or $f \equiv 0$;

- if $\operatorname{deg} f=n$, then $f$ is symmetric and homogeneous in $y_{1}, \ldots, y_{m}$.

\footnotetext{
${ }^{1}$ http://doc.sagemath.org/html/en/reference/combinat/sage/combinat/sf/jack.html
} 
Moreover, any polynomial $f \in V_{n}$ can also be viewed as a polynomial in the eigenvalues of an $m \times m$ symmetric, positive-definite matrix $Y$. Then, the notations $f\left(y_{1}, \ldots, y_{m}\right)$ and $f(Y)$ are considered equivalent. Denote the space of $m \times m$ symmetric, positive-definite matrices by $\operatorname{SPD}(m)$.

\subsection{Definition involving the Wishart distribution}

The following definitions, claims, and properties in this section can be found in [20, pp. 9-22].

Definition 2.2. Define the elementary symmetric polynomial

$$
u_{r}\left(x_{1}, \ldots, x_{m}\right):=\sum_{1 \leq i_{1}<\cdots<i_{r} \leq m} x_{i_{1}} \cdots x_{i_{r}} .
$$

Then, we have a basis for $V_{n}$ : for $\lambda=\left(\lambda_{1}, \ldots, \lambda_{k}\right) \in \mathcal{P}_{n}$, define the polynomials

$$
\mathcal{U}_{\lambda}:=u_{1}^{\lambda_{1}-\lambda_{2}} u_{2}^{\lambda_{2}-\lambda_{3}} \cdots u_{k-1}^{\lambda_{k-1}-\lambda_{k}} u_{k}^{\lambda_{k}} .
$$

Obviously, $\operatorname{deg} \mathcal{U}_{\lambda}=\left(\lambda_{1}-\lambda_{2}\right)+2\left(\lambda_{2}-\lambda_{3}\right)+\cdots+k \lambda_{k}=\lambda_{1}+\cdots+\lambda_{k}=n$. Associate a lexicographical order to $\mathcal{P}_{n}$ as follows: for $\kappa=\left(\kappa_{1}, \ldots, \kappa_{j}\right), \lambda=\left(\lambda_{1}, \ldots, \lambda_{k}\right) \in \mathcal{P}_{n}$,

$$
\kappa>\lambda \quad: \Leftrightarrow \quad \kappa_{1}=\lambda_{1} \wedge \cdots \wedge \kappa_{l-1}=\lambda_{l-1} \wedge \kappa_{l}>\lambda_{l} \text { for some } l .
$$

Then, we can write the basis formed by $\mathcal{U}_{\lambda}$ as a column vector: $\mathcal{U}:=\left(\mathcal{U}_{(n)}, \mathcal{U}_{(n-1,1)}, \ldots, \mathcal{U}_{(1, \ldots, 1)}\right)^{T}$. This subsection presents a definition of $\mathcal{C}_{\lambda}(Y)$ related to the Wishart distribution, defined as follows.

Definition 2.3. Let $X_{\nu \times m}$ be a matrix such that each row is independently drawn from an $m$-variate normal distribution of mean 0 and with covariance matrix $V$, namely,

$$
\left(x_{i}^{1}, \ldots, x_{i}^{m}\right) \sim \mathcal{N}_{m}(0, V) \quad(1 \leq i \leq \nu) .
$$

Then, we say $S:=X^{T} X$ has the Wishart distribution, denoted by $S=X^{T} X \sim W_{m}(V, \nu)$, where $\nu$ is called the degree of freedom.

Remark 2.4. Recall the 1-dimensional case: if $Z_{1}, \ldots, Z_{k} \sim \mathcal{N}(0,1)$ are independent Gaussian distributed, then $Q:=Z_{1}+\cdots+Z_{k} \sim \chi_{k}^{2}$. In other words, the sum of independent Gaussian distributions is chi-square distributed. Therefore, the Wishart distribution can be viewed as a multidimensional generalization of the chi-square distribution.

Define the linear transform $\tau_{\nu}: V_{n} \longrightarrow V_{n}$, for $Y \in \operatorname{SPD}(m)$, by

$$
\left(\tau_{\nu}\left(\mathcal{U}_{\lambda}\right)\right)(Y):=\mathbb{E}_{W}\left[\mathcal{U}_{\lambda}(Y W)\right] \text { for } W \sim W\left(I_{m}, \nu\right) .
$$

As $\mathcal{U}$ forms a basis of $V_{n}, \tau_{\nu}(\mathcal{U}):=\left(\tau_{\nu}\left(\mathcal{U}_{(n)}\right), \tau_{\nu}\left(\mathcal{U}_{(n-1,1)}\right), \ldots, \tau_{\nu}\left(\mathcal{U}_{(1, \ldots, 1)}\right)\right)^{T}$ must be a linear combination of $\mathcal{U}$, denoted by $\tau_{\nu}(\mathcal{U})=T_{\nu} \mathcal{U}$. Properties of the transition matrix $T_{\nu}$ guarantee a diagonalization as $T_{\nu}=\Xi^{-1} \Lambda_{\nu} \Xi$, where

- $\Lambda_{\nu}=\operatorname{diag}\left(2^{n}(\nu / 2)_{\lambda}\right)$, for $\lambda \in \mathcal{P}_{n}$, is the diagonalization of $T_{\nu}$;

- and $\Xi$ is a nonsingular upper triangular matrix, which is uniquely determined up to a (possibly different) multiplicative constant for each row.

Now, we can define the zonal polynomials.

Definition 2.5. For $\lambda=\left(\lambda_{1}, \ldots, \lambda_{k}\right) \in \mathcal{P}_{n}$, the zonal polynomial $\mathcal{Y}_{\lambda}$ is defined by a vector form

$$
\begin{aligned}
\mathcal{Y}=\left(\mathcal{Y}_{(n)}, \mathcal{Y}_{(n-1,1)}, \ldots, \mathcal{Y}_{(1, \ldots, 1)}\right)^{T} & =\Xi \mathcal{U} \\
& =\Xi\left(\mathcal{U}_{(n)}, \mathcal{U}_{(n-1,1)}, \ldots, \mathcal{U}_{(1, \ldots, 1)}\right)^{T},
\end{aligned}
$$

and define $\mathcal{C}_{\lambda}(Y)=d_{\lambda} \mathcal{Y}_{\lambda}(Y)$ by the constants $d_{\lambda}$, given by

$$
d_{\lambda}=\frac{\prod_{i<j}\left(2 \lambda_{i}-2 \lambda_{j}-i+j\right)}{\prod_{i=1}^{k}\left(2 \lambda_{i}+k-i\right) !} \cdot \frac{2^{n} n !}{(2 n) !} .
$$


Example 2.6. For $n=4, \nu=3$, and $m=2$, we compare an exact computation with a Monte-Carlo experiment. Since $m=2$, we only need to consider partitions of 4 with at most 2 parts and get $\mathcal{U}=\left(\left(y_{1}+y_{2}\right)^{4}, y_{1} y_{2}\left(y_{1}+y_{2}\right)^{2}, y_{1}^{2} y_{2}^{2}\right)$.

The map $\tau_{\nu}$ is defined to be an expectation. In order to approximate $\tau_{\nu}(\mathcal{U})(Y)$ numerically, we sample a large number of Wishart matrices $W$. In Mathematica, the command In[1]:= Random Variate[WishartMatrixDistribution[3, IdentityMatrix[2]]] Out[1] $=\{\{3.0965,-0.551265\},\{-0.551265,1.59861\}\}$

randomly generates such a $2 \times 2$ matrix $W$. For simplicity, let $Y=\operatorname{diag}\left(y_{1}, y_{2}\right)$. The eigenvalues of $Y W$ are in general algebraic expressions, but after plugging them into the symmetric polynomials of $\mathcal{U}$ and simplifying, one gets polynomials back. For these simplifications, it is advisable to employ exact arithmetic instead of floating point numbers, and therefore we convert $W$ to have exact rational entries at the very beginning. Averaging over $N=10^{6}$ samples yields the following approximation $\sum_{i=1}^{N} \mathcal{U}\left(Y W_{i}\right) / N$ for the vector $\tau_{\nu}(\mathcal{U})(Y)$ :

$$
\left(\begin{array}{c}
945.715 y_{1}^{4}+1261.66 y_{1}^{3} y_{2}+1347.86 y_{1}^{2} y_{2}^{2}+1258.51 y_{1} y_{2}^{3}+947.094 y_{2}^{4} \\
210.465 y_{1}^{3} y_{2}+299.699 y_{1}^{2} y_{2}^{2}+209.768 y_{1} y_{2}^{3} \\
119.769 y_{1}^{2} y_{2}^{2}
\end{array}\right) .
$$

Now we want to compare this approximate result with the exact one. For this purpose, we proceed "backwards", i.e., we start with the zonal polynomials $\mathcal{C}_{\lambda}(Y)$ (their coefficients are given in Example 3.5. After dividing them with the constants $d_{\lambda}$, we can use (2.1),

$$
\Xi \cdot\left(\begin{array}{c}
\left(y_{1}+y_{2}\right)^{4} \\
y_{1} y_{2}\left(y_{1}+y_{2}\right)^{2} \\
y_{1}^{2} y_{2}^{2}
\end{array}\right)=\left(\begin{array}{c}
4233600 \cdot\left(y_{1}^{4}+y_{2}^{4}+\frac{4}{7}\left(y_{1}^{3} y_{2}+y_{1} y_{2}^{3}\right)+\frac{18}{35} y_{1}^{2} y_{2}^{2}\right) \\
211680 \cdot\left(\frac{24}{7}\left(y_{1}^{3} y_{2}+y_{1} y_{2}^{3}\right)+\frac{16}{7} y_{1}^{2} y_{2}^{2}\right) \\
967680 \cdot y_{1}^{2} y_{2}^{2}
\end{array}\right)
$$

to determine the matrix $\Xi$. As a result, one obtains

$$
\Xi=120960 \cdot\left(\begin{array}{ccc}
35 & -120 & 48 \\
0 & 6 & -8 \\
0 & 0 & 8
\end{array}\right) \quad \text { and } \quad T_{\nu}=\Xi^{-1} \Lambda_{\nu} \Xi=\left(\begin{array}{ccc}
945 & -2520 & 720 \\
0 & 210 & -120 \\
0 & 0 & 120
\end{array}\right) \text {, }
$$

where $\Lambda_{\nu}=\operatorname{diag}(945,210,120)$. Applying this transition matrix to the $\mathcal{U}$-basis, one finally gets

$$
T_{\nu} \mathcal{U}=\left(\begin{array}{c}
945 y_{1}^{4}+1260 y_{1}^{3} y_{2}+1350 y_{1}^{2} y_{2}^{2}+1260 y_{1} y_{2}^{3}+945 y_{2}^{4} \\
210 y_{1}^{3} y_{2}+300 y_{1}^{2} y_{2}^{2}+210 y_{1} y_{2}^{3} \\
120 y_{1}^{2} y_{2}^{2}
\end{array}\right)
$$

and sees that the previous Monte-Carlo simulation delivered quite accurate results.

\subsection{Definition in differential geometry}

Good references for the material in this subsection are [5, 7, 13]. We first recall the Laplace-Beltrami operator on Riemannian manifolds.

Definition 2.7. On a Riemannian manifold $(M, g)$, the Laplace-Beltrami operator on smooth functions $f \in C^{\infty}(M)$ is given by

$$
\Delta f:=(\operatorname{div} \circ \operatorname{grad}) f=\sum_{i, k=1}^{n} \frac{1}{\sqrt{G}} \partial_{k}\left(g^{i k} \sqrt{G} \partial_{i} f\right)
$$

where $n=\operatorname{dim} M,\left(g_{i j}\right)_{n \times n}$ is the metric matrix, and $G:=\operatorname{det}\left(g_{i j}\right)$.

Remark 2.8. When $M=\mathbb{R}^{n}$ and $\left(g_{i j}\right)=I_{n}$, the identity matrix, we have the usual Laplace operator:

$$
\Delta f=\sum_{i=1}^{n} \frac{\partial^{2} f}{\partial x_{i}^{2}} .
$$

Namely, the Laplace-Beltrami operator is the generalization of the Laplace operator on $\mathbb{R}^{n}$. 
Proposition 2.9. Given $X=H Y H^{T} \in \mathrm{SPD}(m)$, for some orthogonal matrix $H \in O(n)$, and $Y=\operatorname{diag}\left(y_{1}, \ldots, y_{n}\right)$ being the diagonalization of $X$, James [7, eq. 3.12] derived the LaplaceBeltrami operator as

$$
\Delta=\sum_{i=1}^{m}\left(y_{i}^{2} \frac{\partial^{2}}{\partial y_{i}^{2}}-\frac{m-3}{2} y_{i} \frac{\partial}{\partial y_{i}}+\sum_{j=1, j \neq i}^{m} \frac{y_{i}^{2}}{y_{i}-y_{j}} \cdot \frac{\partial}{\partial y_{i}}\right)
$$

Remark 2.10. The second term on the right-hand side of 2.2 is, up to the constant $(m-3) / 2$, the Euler's operator $\sum_{i=1}^{m} y_{i} \frac{\partial}{\partial y_{i}}$, which has all symmetric, homogeneous polynomials as its eigenfunctions. Thus, when considering eigenfunctions of the Laplace-Beltrami operator, it can be eliminated.

Definition 2.11. The zonal polynomials $\mathcal{C}_{\lambda}\left(y_{1}, \ldots, y_{m}\right)$ are the eigenfunctions of the operator $\Delta_{Y}$, defined by

$$
\Delta_{Y}:=\sum_{i=1}^{m}\left(y_{i}^{2} \frac{\partial^{2}}{\partial y_{i}^{2}}+\sum_{j=1, j \neq i}^{m} \frac{y_{i}^{2}}{y_{i}-y_{j}} \cdot \frac{\partial}{\partial y_{i}}\right) .
$$

In particular, for $\lambda=\left(\lambda_{1}, \ldots, \lambda_{k}\right) \in \mathcal{P}_{n}$, we have

$$
\Delta_{Y} \mathcal{C}_{\lambda}(Y)=\left(\rho_{\lambda}+(m-1) n\right) \cdot \mathcal{C}_{\lambda}(Y)
$$

where

$$
\rho_{\lambda}:=\sum_{i=1}^{k} \lambda_{i}\left(\lambda_{i}-i\right)
$$

\subsection{Definition through representation theory}

Consider the general linear group $G=\mathrm{GL}(m)$ on $V_{n}$. Define a representation as follows. For $g \in \mathrm{GL}(m), Y \in V_{n}$, and $\varphi \in \mathrm{GL}\left(V_{n}\right)$,

$$
(g \circ \varphi)(Y):=\varphi\left(g^{-1} Y\left(g^{-1}\right)^{T}\right) .
$$

As a representation, the linear space can be decomposed into invariant subspaces [6. p. 611]

$$
V_{n}=\bigoplus_{\lambda \in \mathcal{P}_{n}} V_{\lambda}
$$

Definition 2.12. Given $Y \in \operatorname{SPD}(m)$ and $\lambda \in \mathcal{P}_{n}$, define the zonal polynomials by the projection

$$
\mathcal{C}_{\lambda}(Y)=\left.(\operatorname{tr} Y)^{n}\right|_{V_{\lambda}} .
$$

Remark 2.13. Note that 2.4 confirms (1.3).

\subsection{A short remark on Macdonald, Jack and zonal polynomials}

A limit case (by taking $t=q^{\alpha}$ and letting $q \rightarrow 1$ ) of the Macdonald polynomials gives the Jack polynomials $J_{\lambda}^{(\alpha)}$, which when $\alpha=2$, gives the zonal polynomials $\mathcal{Z}_{\lambda}(Y) . \mathcal{Z}_{\lambda}(Y)$ differs from $\mathcal{C}_{\lambda}(Y)$ only by a constant factor.

\section{Calculation of zonal polynomials}

Although there are several ways to define the zonal polynomial $\mathcal{C}_{\lambda}(Y)$, in practice, none of these definitions gives an algorithm or formula to directly compute $\mathcal{C}_{\lambda}(Y)$. Now, we follow the steps by Muirhead [15] to build up packages for the calculation of $\mathcal{C}_{\lambda}(Y)$. 
Definition 3.1. For $\lambda=\left(\lambda_{1}, \ldots, \lambda_{k}\right) \in \mathcal{P}_{n}$, define the monomial symmetric function as

$$
M_{\lambda}\left(y_{1}, \ldots, y_{m}\right)=\sum_{\substack{i_{1}, \ldots, i_{k} \\ \text { distinct terms }}} y_{i_{1}}^{\lambda_{1}} \cdots y_{i_{k}}^{\lambda_{k}}=y_{1}^{\lambda_{1}} \cdots y_{k}^{\lambda_{k}}+\text { symmetric terms. }
$$

Remark 3.2. An explicit expression of $M_{\lambda}(Y)$ is given by [20, eq. 6]:

$$
M_{\left(1^{m_{1}} 2^{\left.m_{2} \ldots\right)}\right)}(Y)=\left(\prod_{j=1}^{n} \frac{1}{m_{j} !}\right) \sum_{i_{1}, \ldots, i_{k}} y_{i_{1}}^{\lambda_{1}} \cdots y_{i_{k}}^{\lambda_{k}},
$$

where $\lambda=\left(\lambda_{1}, \ldots, \lambda_{k}\right)=\left(1^{m_{1}}, 2^{m_{2}}, \ldots, n^{m_{n}}\right) \in \mathcal{P}_{n}$ contains $m_{1} 1$ 's, $m_{2} 2$ 's, etc.

Theorem 3.3. We have, for some constants $c_{\kappa, \lambda}$, that (see, e.g., [15], eq. 13])

$$
\mathcal{C}_{\kappa}(Y)=\sum_{\lambda \leq \kappa} c_{\kappa, \lambda} M_{\lambda}(Y)
$$

Remark 3.4. Note that $M_{\lambda}(Y)$ is defined to be zero whenever there are fewer variables than parts in the partition $\lambda$. It follows that $\mathcal{C}_{\kappa}(Y)=0$ if the dimension of $Y$ is less than the number of parts of $\kappa$.

Example 3.5. The following table, from [15, p. 238], shows the coefficients $c_{\kappa, \lambda}$, in the case $n=4$.

\begin{tabular}{c|ccccc}
$\kappa \backslash \lambda$ & $(4)$ & $(3,1)$ & $(2,2)$ & $(2,1,1)$ & $(1,1,1,1)$ \\
\hline$(4)$ & 1 & $\frac{4}{7}$ & $\frac{18}{35}$ & $\frac{12}{35}$ & $\frac{8}{35}$ \\
$(3,1)$ & 0 & $\frac{24}{7}$ & $\frac{16}{7}$ & $\frac{88}{21}$ & $\frac{32}{7}$ \\
$(2,2)$ & 0 & 0 & $\frac{16}{5}$ & $\frac{32}{15}$ & $\frac{16}{5}$ \\
$(2,1,1)$ & 0 & 0 & 0 & $\frac{16}{3}$ & $\frac{64}{5}$ \\
$(1,1,1,1)$ & 0 & 0 & 0 & 0 & $\frac{16}{5}$
\end{tabular}

Theorem 3.6. The constant $c_{\kappa, \lambda}$ satisfies the recurrence [15, eq. 14]

$$
c_{\kappa, \lambda}=\sum_{\lambda<\mu \leq \kappa} \frac{\left(\lambda_{r}+t\right)-\left(\lambda_{s}-t\right)}{\rho_{\kappa}-\rho_{\lambda}} c_{\kappa, \mu},
$$

where the sum is over all $\mu=\left(\lambda_{1}, \ldots, \lambda_{r-1}, \lambda_{r}+t, \lambda_{r+1}, \ldots, \lambda_{s-1}, \lambda_{s}-t, \lambda_{s+1}, \ldots, \lambda_{k}\right)$, for $\lambda=\left(\lambda_{1}, \ldots, \lambda_{k}\right)$ and $t=1, \ldots, \lambda_{s}$ such that by rearranging the tuple $\mu$ in a descending order, it lies as $\lambda<\mu \leq \kappa$. Recall the quantity $\rho_{\kappa}$ that is defined in 2.3.

Remark 3.7. As mentioned in Remark 1 on page 73 of [20], recurrence (3.4) fails when $\rho_{\kappa}-\rho_{\lambda}=0$, which first occurs when $\kappa=(4,1,1)$ and $\lambda=(3,3)$ :

$$
\rho_{\kappa}=4 \cdot(4-1)+1 \cdot(1-2)+1 \cdot(1-3)=9=3 \cdot(3-1)+3 \cdot(3-2)=\rho_{\lambda} .
$$

In this case, not only does the denominator in (3.4) vanish, but also the summation is empty. It seems that James [7] has claimed that for all "relevant" pairs $(\kappa, \lambda), c_{\kappa, \lambda}>0$. Due to the nonnegative numerator of the summation in (3.4), it suggests that if $\rho_{\kappa} \leq \rho_{\lambda}, c_{\kappa, \lambda}=0$.

See the example at the end of Section 8 of the case $\kappa=(4,1,1)$ and $\lambda=(3,3)$, which is also compatible with the built-in package in SageMath.

Once the initial value $c_{\kappa, \kappa}$ is given, (3.4) can compute $c_{\kappa, \lambda}$ for all $\lambda<\kappa$. Now, observing the tables in Example 3.5 and recalling [1.3, it is easy to see that the sum of each column is given by a multinomial coefficient. More precisely, let $\lambda=\left(\lambda_{1}, \ldots, \lambda_{k}\right) \in \mathcal{P}_{n}$,

$$
\sum_{\kappa=\lambda}^{(n)} c_{\kappa, \lambda}=\left(\begin{array}{c}
n \\
\lambda_{1}, \ldots, \lambda_{k}
\end{array}\right)
$$

In particular, $c_{(n),(n)}=\left(\begin{array}{l}n \\ n\end{array}\right)=1$. Thus, all constants $c_{\kappa, \lambda}$ are obtained, and so is $\mathcal{C}_{\lambda}(Y)$. 


\section{Characterization of vanishing coefficients}

Muirhead [15, Lem. 7.2.3] gives a necessary condition for some coefficients $c_{\kappa, \lambda}$ to be zero, but without a proof. We recall his result here and give a simple proof of it.

Lemma 4.1. Let $n \in \mathbb{N}$ and $\kappa, \lambda \in \mathcal{P}_{n}$. If $\kappa$ has more parts than $\lambda$, then $c_{\kappa, \lambda}=0$.

Proof. Given a partition $\lambda$, denote by len $(\lambda)$ its number of parts. Assume that len $(\kappa)=k$. Then, the definition of zonal polynomials 3.3 and Remark 3.4 , we see that

$$
\begin{aligned}
0 & =\mathcal{C}_{\kappa}\left(y_{1}, \ldots, y_{k-1}\right) \\
& =\sum_{\substack{\lambda \leq \kappa \\
\operatorname{len}(\lambda)<k}} c_{\kappa, \lambda} \underbrace{M_{\lambda}\left(y_{1}, \ldots, y_{k-1}\right)}_{\neq 0}+\sum_{\begin{array}{c}
\lambda \leq \kappa \\
\operatorname{len}(\lambda) \geq k
\end{array}} c_{\kappa, \lambda} \underbrace{M_{\lambda}\left(y_{1}, \ldots, y_{k-1}\right)}_{=0} .
\end{aligned}
$$

Now, it is apparent that all coefficients $c_{\kappa, \lambda}$ for which $\lambda$ has fewer parts than $k$ must be zero.

While Lemma 4.1 only gives a necessary condition under which $c_{\kappa, \lambda}$ is zero, we would like to obtain a full characterization, i.e., a necessary and sufficient condition for $c_{\kappa, \lambda}=0$. For example, we have $c_{(8,2,2),(7,4,1)}=0$, although both partitions have the same length.

Theorem 4.2. Let $n \in \mathbb{N}$ and let $\kappa, \lambda \in \mathcal{P}_{n}$ with $\kappa \geq \lambda$ in lexicographic order. Then $c_{\kappa, \lambda}=0$ if and only if there exists $p \in \mathbb{N}$ such that, (where the partitions are filled with zeros as necessary,)

$$
\sum_{i=1}^{p}\left(\kappa_{i}-\lambda_{i}\right)<0 .
$$

Proof. We first want to show that, under the assumption 4.1), $\sum_{i=1}^{p}\left(\kappa_{i}-\mu_{i}\right)<0$ holds for all $\mu$ of the form $\mu=\left(\ldots, \lambda_{r}+t, \ldots, \lambda_{s}-t, \ldots\right), 1 \leq t \leq \lambda_{s}$, after reordering the parts. Assume that this reordering requires us to move the part $\lambda_{r}+t$ to the $k$-th position $(k \leq r)$ and the part $\lambda_{s}-t$ to the $\ell$-th position $(\ell \geq s)$. Then the partition $\mu$ has the following form:

$$
\left(\lambda_{1}, \ldots, \lambda_{k-1}, \lambda_{r}+t, \lambda_{k}, \ldots, \lambda_{r-1}, \lambda_{r+1}, \ldots, \lambda_{s-1}, \lambda_{s+1}, \ldots, \lambda_{\ell}, \lambda_{s}-t, \lambda_{\ell+1}, \ldots\right) .
$$

Then we have

$$
\sum_{i=1}^{p} \mu_{i}= \begin{cases}\sum_{i=1}^{p} \lambda_{i}, & \text { if } p<k ; \\ \sum_{i=1}^{p-1} \lambda_{i}+\lambda_{r}+t, & \text { if } k \leq p<r ; \\ \sum_{i=1}^{p} \lambda_{i}+t, & \text { if } r \leq p<s \\ \sum_{i=1}^{s-1} \lambda_{i}+t+\sum_{i=s+1}^{p+1} \lambda_{i}, & \text { if } s \leq p<\ell ; \\ \sum_{i=1}^{p} \lambda_{i}, & \text { if } p \geq \ell .\end{cases}
$$

In each of these cases, we claim $\sum_{i=1}^{p} \mu_{i} \geq \sum_{i=1}^{p} \lambda_{i}$. For the 1 st, 3 rd and 5 th case, it is immediately obvious. For the 2nd and 4 th case, it is true because $\lambda_{r}+t \geq \lambda_{p}$ and $\lambda_{p+1} \geq \lambda_{s}-t$, respectively.

We have just shown that if $\kappa$ and $\lambda$ satisfy (4.1), then all the $\mu$ 's in (3.4) also satisfy (4.1), in other words, $c_{\kappa, \lambda}$ is a linear combination of $c_{\kappa, \mu}$ 's with the same property. In order to conclude the proof by induction, we have to investigate the two possible base cases:

1. We arrive at a $c_{\kappa, \lambda}$ for which $\lambda$ has fewer parts than $\kappa$ (, note that the operation $\left(\ldots, \lambda_{r}+\right.$ $\left.t, \ldots, \lambda_{s}-t, \ldots\right)$ weakly decreases the number of parts). Then by Lemma 4.1. $c_{\kappa, \lambda}=0$.

2. We arrive at a $c_{\kappa, \lambda}$ such that no suitable $\mu$ between $\lambda$ and $\kappa$ exists. Also in this case we get $c_{\kappa, \lambda}=0$ since the sum in 3.4 is empty.

Now we show the converse: if $\kappa, \lambda$ do not satisfy (4.1), then by (3.4), $c_{\kappa, \lambda}$ is a linear combination (with nonnegative coefficients) of $c$ 's that do not satisfy (4.1) either. Note that $\rho_{\kappa}<\rho_{\lambda}$ only 
occurs if 4.1 holds, which can be proven as follows. Let $\kappa=\left(\kappa_{1}, \ldots, \kappa_{m}\right)$ and $\lambda=\left(\lambda_{1}, \ldots, \lambda_{m}\right)$, with possible zero components as necessary. For any constant $c$,

$$
\begin{aligned}
\rho_{\kappa}-\rho_{\lambda} & =\sum_{i=1}^{m}\left(\kappa_{i}\left(\kappa_{i}-i\right)-\lambda_{i}\left(\lambda_{i}-i\right)\right) \\
& =\sum_{i=1}^{m}\left(\kappa_{i}-\lambda_{i}\right)\left(\kappa_{i}+\lambda_{i}-i\right) \\
& =\sum_{i=1}^{m}\left(\kappa_{i}-\lambda_{i}\right)\left(\kappa_{i}+\lambda_{i}-i+c\right)-c \sum_{i=1}^{m}\left(\kappa_{i}-\lambda_{i}\right) \\
& =\sum_{i=1}^{m}\left(\kappa_{i}-\lambda_{i}\right)\left(\kappa_{i}+\lambda_{i}-i+c\right),
\end{aligned}
$$

due to that

$$
\sum_{i=1}^{m}\left(\kappa_{i}-\lambda_{i}\right)=\sum_{i=1}^{m} \kappa_{i}-\sum_{i=1}^{k} \lambda_{i}=n-n=0 .
$$

Let $c=m+1$ and define $a_{i}=\kappa_{i}+\lambda_{i}+(m+1-i)$ for $i=1,2, \ldots, m$, which are positive and strictly decreasing. If $\rho_{\kappa}<\rho_{\lambda}$ but (4.1) fails, i.e., for any $p \in \mathbb{N}, \sum_{i=1}^{p}\left(\kappa_{i}-\lambda_{i}\right) \geq 0$, we see the contradiction as follows.

$$
\begin{aligned}
\rho_{\kappa}-\rho_{\lambda}= & \sum_{i=1}^{m}\left(\kappa_{i}-\lambda_{i}\right) a_{i} \\
= & a_{m} \sum_{i=1}^{m}\left(\kappa_{i}-\lambda_{i}\right)+\left(a_{m-1}-a_{m}\right) \sum_{i=1}^{m-1}\left(\kappa_{i}-\lambda_{i}\right)+\cdots \\
& \quad+\left(a_{1}-a_{2}\right)\left(\kappa_{1}-\lambda_{1}\right) \geq 0 .
\end{aligned}
$$

Thus, we only focus on the case that $\rho_{\kappa}>\rho_{\lambda}$. It is not difficult to see that $\lambda$ can be converted into $\kappa$ by a finite sequence of moves $\left(\ldots, \lambda_{r}+1, \lambda_{r+1}-1, \ldots\right)$, where $r$ is the smallest index such that $\sum_{i=1}^{r}\left(\kappa_{i}-\lambda_{i}\right)>0$, and all partitions in this sequence do not satisfy 4.1. The recursion ends when there is no such $r$, i.e., when $\lambda=\kappa$, and one sees that the contribution of $c_{\kappa, \kappa} \neq 0$ makes $c_{\kappa, \lambda}$ positive.

Example 4.3. For $\kappa=(8,2,2)$ and $\lambda=(7,4,1)$ we verify that the second partial sum of their differences, i.e., $p=2$ is negative: $(8-7)+(2-4)=-1$, and hence $c_{\kappa, \lambda}$ must be zero.

A graphical presentation of the positions where $c_{\kappa, \lambda}=0$ is shown in Figure 4.1. We recognize a fractal-like structure which is due to Theorem 4.2 and the lexicographical ordering of partitions.

\section{Infinite families of coefficients $c_{\kappa, \lambda}$}

In this section, we study some infinite families among the coefficients $c_{\kappa, \lambda}$ of the zonal polynomials $\mathcal{C}_{\kappa}(Y)$. Since we have seen that $c_{(n),(n)}=1$ for all $n$ (that corresponds to the upper left corner of the $c_{\kappa, \lambda}$-matrix), one can ask whether the "neighboring" entries also admit a closed form for general $n$. Theorem 5.2 will give an explicit answer for the first few cases. Before that, we focus on the first row of the $c_{\kappa, \lambda}$-matrix, i.e., on the coefficients $c_{\kappa, \lambda}$ for which $\kappa=(n)$ and $\lambda \leq \kappa$ has two parts.

Theorem 5.1. Let $(n-m, m) \in \mathcal{P}_{n}$, then

$$
c_{(n),(n-m, m)}=\left(\begin{array}{c}
n \\
m
\end{array}\right) \cdot \frac{\left(\frac{1}{2}\right)_{m}}{\left(n-m+\frac{1}{2}\right)_{m}} .
$$




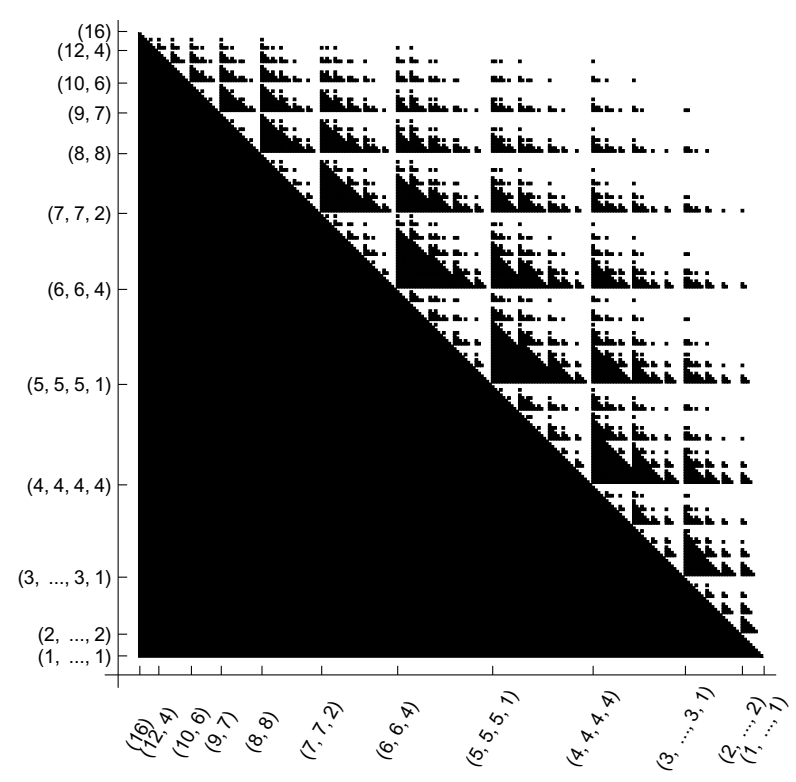

FIGURE 4.1. Location of zeros in the $c_{\kappa, \lambda}$-matrix for $n=16$ : each nonzero coefficient is represented by a white square, each zero by a black square.

Proof. Obviously, $\rho_{(n)}-\rho_{(n-m, m)}=m(2 n-2 m+1)$. By (3.4),

$$
\begin{aligned}
c_{(n),(n-m, m)} & =\sum_{(n-m, m)<\mu \leq(n)} \frac{(n-m+t)-(m-t)}{\rho_{(n)}-\rho_{(n-m, m)}} \cdot c_{(n), \mu} \\
& =\frac{1}{m(2 n-2 m+1)} \sum_{t=1}^{m}(n-2 m+2 t) c_{(n), \mu} .
\end{aligned}
$$

Now, we proceed by induction on $m$. When $m=1$, the only term in the sum is $t=1$, i.e., $\mu=(n)$, so that

$$
c_{(n),(n-1,1)}=\frac{1}{2 n-1} \cdot(n-2+2) \cdot 1=\frac{n}{2 n-1}=\left(\begin{array}{c}
n \\
1
\end{array}\right) \frac{\frac{1}{2}}{n-\frac{1}{2}} .
$$

Now, assume (5.1) holds for $m$. Considering the case $m+1$, we have

$$
\begin{aligned}
& c_{(n),(n-m-1, m+1)}= \\
& =\frac{1}{(m+1)(2(n-m)-1)} \sum_{t=1}^{m+1}(n-2 m-2+2 t) c_{(n), \mu} \\
& =\frac{1}{(m+1) 2(n-m)-1)}\left((n-2 m) c_{(n),(n-m, m)}+\sum_{t=1}^{m}(n-2 m+2 t) c_{(n), \mu}\right) \\
& =\frac{c_{(n),(n-m, m)}}{(m+1)(2(n-m)-1)}((n-2 m)+m(2(n-m)+1)) \\
& =\frac{(n-m)(2 m+1)}{(m+1)(2(n-m)-1)} \cdot\left(\begin{array}{c}
n \\
m
\end{array}\right) \frac{\left(\frac{1}{2}\right)_{m}}{\left(n-m+\frac{1}{2}\right)_{m}} \\
& =\left(\begin{array}{c}
n \\
m+1
\end{array}\right) \cdot \frac{\left(\frac{1}{2}\right)_{m+1}}{\left(n-m-\frac{1}{2}\right)_{m+1}} .
\end{aligned}
$$


Theorem 5.2. We have

$$
\begin{aligned}
c_{(n-1,1),(n-1,1)} & =\frac{2 n(n-1)}{2 n-1}, & & (n \geq 2) ; \\
c_{(n-1,1),(n-2,2)} & =\frac{2 n(n-1)(n-2)}{(2 n-1)(2 n-5)}, & & (n \geq 4) ; \\
c_{(n-2,2),(n-2,2)} & =\frac{2 n(n-1)(n-2)(n-3)}{(2 n-3)(2 n-5)}, & & (n \geq 4) .
\end{aligned}
$$

Proof. Using (5.2) and (3.5) with $\lambda=(n-1,1)$, we have

$$
\begin{aligned}
c_{(n-1,1),(n-1,1)} & =\left(\begin{array}{c}
n \\
n-1,1
\end{array}\right)-c_{(n),(n-1,1)} \\
& =n-\frac{n}{2 n-1}=\frac{2 n^{2}-2 n}{2 n-1}=\frac{2 n(n-1)}{2 n-1} .
\end{aligned}
$$

By applying (3.4), we obtain

$$
\begin{aligned}
c_{(n-1,1),(n-2,2)} & =\frac{(n-2+1)-(2-1)}{\rho_{(n-1,1)}-\rho_{(n-2,2)}} \cdot c_{(n-1,1),(n-1,1)} \\
& =\frac{(n-2+1)-(2-1)}{2 n-5} \cdot \frac{2 n(n-1)}{2 n-1}=\frac{2 n(n-1)(n-2)}{(2 n-1)(2 n-5)} .
\end{aligned}
$$

Finally, using (3.5) and (5.1) with $\lambda=(n-2,2)$, we get the last coefficient:

$$
\begin{aligned}
c_{(n-2,2),(n-2,2)} & =\left(\begin{array}{c}
n \\
n-2,2
\end{array}\right)-c_{(n),(n-2,2)}-c_{(n-1,1),(n-2,2)} \\
& =\frac{2 n(n-1)(n-2)(n-3)}{(2 n-3)(2 n-5)} .
\end{aligned}
$$

It is clear that we could proceed in this manner and compute more coefficients $c_{\kappa, \lambda}$ in the upper left corner of the matrix for symbolic $n$. Since the corresponding calculations get too tedious to be done by hand, we employ computer algebra to determine the rational function expressions for a few more coefficients. Note that, when $n$ is sufficiently large, the lexicographically largest elements of $\mathcal{P}_{n}$ are (in descending order):

$$
(n),(n-1,1),(n-2,2),(n-2,1,1),(n-3,3),(n-3,2,1), \text { etc. }
$$

In Table 1 we give closed forms for the coefficients $c_{\kappa, \lambda}$ when $\kappa$ and $\lambda$ are taken from these lexicographically largest partitions, i.e., when both $\kappa$ and $\lambda$ are of the form $(n-m, \pi)$, where $\pi \in \mathcal{P}_{m}$ but $n$ is symbolic. Pictorially speaking, this table represents the upper left submatrix of the $c_{\kappa, \lambda}$-matrix for large $n$; more precisely, for $n \geq 6$ since we consider only partitions $(n), \ldots,(n-3,1,1,1)$. We remark that all these formulas have been rigorously proven by applying (3.4) and (3.5) for symbolic $n$, as it was done in the proof of Theorem 5.2. This symbolic proof strategy is implemented in our Mathematica package as ZonalCoefficientN (see Section 9).

Example 5.3. For example, for $n=23$ we obtain the coefficient $c_{(21,2),(21,1,1)}$ by reading the entry in Table 1 in row $(n-2,2)$ and column $(n-2,1,1)$ :

$$
c_{(21,2),(21,1,1)}=\left.\frac{4(n-3)(n-2)(n-1) n}{3(2 n-5)(2 n-3)}\right|_{n=23}=\frac{4 \cdot 20 \cdot 21 \cdot 22 \cdot 23}{3 \cdot 41 \cdot 43}=\frac{283360}{1763} .
$$

We formulate a conjecture on the limit, as $n$ goes to infinity, of the coefficients of the form $c_{(n),\left(n-n^{\prime}, \lambda^{\prime}\right)}$ 


\begin{tabular}{c|cccc}
$\kappa \backslash \lambda$ & $(n)$ & $(n-1,1)$ & $(n-2,2)$ & $(n-2,1,1)$ \\
\hline$(n)$ & 1 & $\frac{n}{2 n-1}$ & $\frac{3(n-1) n}{2(2 n-3)(2 n-1)}$ & $\frac{(n-1) n}{(2 n-3)(2 n-1)}$ \\
$(n-1,1)$ & 0 & $\frac{2(n-1) n}{2 n-1}$ & $\frac{2(n-2)(n-1) n}{(2 n-5)(2 n-1)}$ & $\frac{2 n\left(2 n^{2}-6 n+3\right)}{(2 n-5)(2 n-1)}$ \\
$(n-2,2)$ & 0 & 0 & $\frac{2(n-3)(n-2)(n-1) n}{(2 n-5)(2 n-3)}$ & $\frac{4(n-3)(n-2)(n-1) n}{3(2 n-5)(2 n-3)}$ \\
$(n-2,1,1)$ & 0 & 0 & 0 & $\frac{2}{3}(n-2) n$ \\
$\kappa \backslash \lambda$ & & $(n-3,3)$ & $\frac{(n-3,2,1)}{2(2 n-5)(2 n-3)(2 n-1)}$ \\
$(n)$ & $\frac{5(n-2)(n-1) n}{2(2 n-5)(2 n-3)(2 n-1)}$ & $\frac{(n-2) n\left(5 n^{2}-20 n+11\right)}{(2 n-7)(2 n-5)(2 n-1)}$ \\
$(n-1,1)$ & $\frac{3(n-3)(n-2)(n-1) n}{(2 n-7)(2 n-5)(2 n-1)}$ & $\frac{2(n-3)(n-1) n\left(5 n^{2}-30 n+36\right)}{3(2 n-9)(2 n-5)(2 n-3)}$ \\
$(n-2,2)$ & $\frac{2(n-4)(n-3)(n-2)(n-1) n}{(2 n-9)(2 n-5)(2 n-3)}$ & $\frac{2(n-3)(n-2) n}{3(2 n-7)}$ \\
$(n-2,1,1)$ & 0 & $\frac{4(n-5)(n-4)(n-3)(n-2)(n-1) n}{5(2 n-9)(2 n-7)(2 n-5)}$ \\
$(n-3,3)$ & $\frac{4(n-5)(n-4)(n-3)(n-2)(n-1) n}{3(2 n-9)(2 n-7)(2 n-5)}$ & $\frac{4(n-4)(n-3)(n-1) n}{5(2 n-7)}$
\end{tabular}

TABLE 1. Coefficients $c_{\kappa, \lambda}$ for some of the lexicographically largest partitions of $n$; the lower table continues the upper one to the right.

Conjecture 5.4. Let $n^{\prime}<n$ and $\lambda^{\prime}=\left(p_{1}, \ldots, p_{\ell}\right) \in \mathcal{P}_{n^{\prime}}$. Then,

$$
\lim _{n \rightarrow \infty} c_{(n),\left(n-n^{\prime}, \lambda^{\prime}\right)}=\prod_{i=1}^{\ell} \frac{\left(p_{i}\right)_{p_{i}}}{p_{i} ! 2^{2 p_{i}-1}}
$$

Example 5.5. Let $n^{\prime}=3$ and $\lambda^{\prime}=(2,1)$. By Table 1 and the conjecture above

$$
\begin{aligned}
\lim _{n \rightarrow \infty} c_{(n),(n-3,2,1)} & =\lim _{n \rightarrow \infty} \frac{3(n-2)(n-1) n}{2(2 n-5)(2 n-3)(2 n-1)}=\frac{3}{16} \\
& =\frac{(2)_{2}}{2 ! 2^{2 \cdot 2-1}} \cdot \frac{(1)_{1}}{1 ! 2^{2 \cdot 1-1}} .
\end{aligned}
$$

The next families we study are located in the "lower right corner" of $c_{\kappa, \lambda}$-matrix. In this case both $\kappa$ and $\lambda$ are of the form $\left(2^{m}, 1^{n-2 m}\right)$, i.e., a sequence of $m$ times the part 2 and $n-2 m$ times the part 1 . Note that for $m=0,1,2, \ldots$ we obtain the lexicographically smallest partitions of $n$ (in increasing order). In contrast to Theorem 5.2 it is not straightforward to derive formulas for general $n$ by means of Equations (3.4) and (3.5). Instead, we compute the first few values of these sequences, say up to $n=30$, and then guess a closed form expression. Some results are displayed in Table 2

More precisely, we first applied the Guess .m package [9] to find a plausible candidate for a linear recurrence with polynomial coefficients (in all considered instances this recurrence was of first order, which easily allowed for a closed form solution). Then, after observing that all expressions obtained this way were of the form $2^{n} \cdot r(n)$ where $r(n)$ is some rational function in $n$, we refined our ansatz to only search for expressions of this form: divide the $n$-th sequence entry by $2^{n}$ and then perform polynomial interpolation and rational reconstruction. 


\begin{tabular}{c|ccc}
$\kappa \backslash \lambda$ & $\left(2^{4}, 1^{n-8}\right)$ & $\left(2^{3}, 1^{n-6}\right)$ & $\left(2^{2}, 1^{n-4}\right)$ \\
\hline$\left(2^{4}, 1^{n-8}\right)$ & $\frac{2^{n-3}(n-6)(n-5) n}{15}$ & $\frac{2^{n-3}(n-7)(n-6)^{2} n}{15}$ & $\frac{2^{n-4}(n-7)(n-6)^{2}(n-5) n}{15}$ \\
$\left(2^{3}, 1^{n-6}\right)$ & 0 & $\frac{2^{n-3}(n-4)(n-3)}{3}$ & $\frac{2^{n-3}(n-5)(n-4)^{2}}{3}$ \\
$\left(2^{2}, 1^{n-4}\right)$ & 0 & 0 & $\frac{2^{n-1}(n-2)(n-1)}{3(n+1)}$ \\
$\kappa \backslash \lambda$ & $\left(2,1^{n-2}\right)$ & $\left(1^{n}\right)$ \\
\hline$\left(2^{4}, 1^{n-8}\right)$ & $\frac{2^{n-4}(n-7)(n-6)^{2}(n-5)(n-2) n}{45}$ & $\frac{2^{n-6}(n-7)(n-6)^{2}(n-5)(n-1) n^{2}}{45}$ \\
$\left(2^{3}, 1^{n-6}\right)$ & $\frac{2^{n-4}(n-5)(n-4)^{2}(n-3)}{3}$ & $\frac{2^{n-4}(n-5)(n-4)^{2}(n-3) n}{9}$ \\
$\left(2^{2}, 1^{n-4}\right)$ & $\frac{2^{n-1}(n-3)(n-2)^{2}}{3(n+1)}$ & $\frac{2^{n-2}(n-3)(n-2)^{2}(n-1)}{3(n+1)}$ \\
$\left(2,1^{n-2}\right)$ & $\frac{2^{n-1} n}{n+2}$ & $\frac{2^{n-1}(n-1) n^{2}}{(n+1)(n+2)}$ \\
$\left(1^{n}\right)$ & 0 & $\frac{2^{n}}{n+1}$
\end{tabular}

TABLE 2. Coefficients $c_{\kappa, \lambda}$ for some of the lexicographically smallest partitions of $n$; the lower table continues the upper one to the right.

\section{Partitions with two parts}

Now we concentrate on the coefficients $c_{\kappa, \lambda}$ when both $\kappa$ and $\lambda$ have at most two parts. Takemura [20, $\S 4.4]$ gives formulas for these coefficients in terms of elementary symmetric functions, while we focus on monomial symmetric functions.

When applying the recursive formula (3.4) one sees that the partitions $\mu$ cannot have more parts than $\lambda$, by the way how they are constructed. Similarly, when we use (3.5) to compute $c_{\lambda, \lambda}$, only those coefficients $c_{\kappa, \lambda}$ contribute for which $\kappa$ has at most two parts; this is a direct consequence of Lemma 4.1 We conclude that, for the computation of $c_{\kappa, \lambda}$, we do not need any $c_{\kappa^{\prime}, \lambda^{\prime}}$ with $\kappa^{\prime}$ or $\lambda^{\prime}$ having more than two parts. Throughout this section we write

$$
\kappa=(a, a-b) \quad \text { and } \quad \lambda=(a-d, a-b+d)
$$

for nonnegative integers $a, b, d$. The condition $b<a$ ensures that $\kappa$ is a proper partition and the condition $d \leq b / 2$ ensures that the parts of $\lambda$ are in the correct order. In Theorem 6.3 we present a closed form of the coefficients $c_{\kappa, \lambda}$ under these assumptions. We start our investigation by specializing (3.4) and (3.5) to partitions with two parts. First of all, it is easy to compute that

$$
\rho_{\kappa}-\rho_{\lambda}=d \cdot(2 b-2 d+1) .
$$

Then, recurrence (3.4) can be written as, by noting that $\lambda$ has only two parts,

$$
\begin{aligned}
c_{\kappa, \lambda}=c_{(a, a-b),(a-d, a-b+d)} & =\sum_{\lambda<\mu \leq \kappa} \frac{(a-d+t)-(a-b+d-t)}{\rho_{\kappa}-\rho_{\lambda}} c_{\kappa, \mu} \\
& =\sum_{t=1}^{d} \frac{b-2 d+2 t}{d(2 b-2 d+1)} c_{(a, a-b),(a-d+t, a-b+d-t)} \\
& =\sum_{j=0}^{d-1} \frac{b-2 j}{d(2 b-2 d+1)} c_{(a, a-b),(a-j, a-b+j)} .
\end{aligned}
$$


Example 6.1. We consider some specific examples. For $d=1,2,3$, recursively applying (6.1) yields

$$
\begin{aligned}
& c_{(a, a-b),(a-1, a-b+1)}=\frac{b}{2 b-1} c_{(a, a-b),(a, a-b)}, \\
& c_{(a, a-b),(a-2, a-b+2)}=\frac{3 b(b-1)}{2(2 b-1)(2 b-3)} c_{(a, a-b),(a, a-b)}, \\
& c_{(a, a-b),(a-3, a-b+3)}=\frac{5 b(b-1)(b-2)}{2(2 b-1)(2 b-3)(2 b-5)} c_{(a, a-b),(a, a-b)} .
\end{aligned}
$$

Proposition 6.2. Given two partitions $\kappa=(a, a-b)$ and $\lambda=(a-d, a-b+d)$ of the positive integer $n=2 a-b$, with $0 \leq b<a$ and $0 \leq d \leq b / 2$, then

$$
c_{\kappa, \lambda}=c_{(a, a-b),(a-d, a-b+d)}=\left(\begin{array}{l}
b \\
d
\end{array}\right) \frac{\left(\frac{1}{2}\right)_{d}}{\left(b-d+\frac{1}{2}\right)_{d}} \cdot c_{(a, a-b),(a, a-b)} .
$$

Proof. Note that this result is a direct generalization of Theorem 5.1. and hence the same inductive argument could be applied. Instead, we illustrate a different proof strategy, using symbolic summation. Because of the recursive definition of the coefficients $c_{\kappa, \lambda}$, demonstrated in Example 6.1, it suffices to show that the asserted expression satisfies the recurrence 6.1]. Namely, we need to prove

$$
\left(\begin{array}{l}
b \\
d
\end{array}\right) \frac{\left(\frac{1}{2}\right)_{d}}{\left(b-d+\frac{1}{2}\right)_{d}}=\sum_{j=0}^{d-1}\left(\begin{array}{l}
b \\
j
\end{array}\right) \frac{(b-2 j)\left(\frac{1}{2}\right)_{j}}{d(2 b-2 d+1)\left(b-j+\frac{1}{2}\right)_{j}} .
$$

Using special-purpose computer algebra packages, such as the HolonomicFunctions package [11], we find that the expression in the sum, denote it by $f(j)$, is Gosper-summable. More precisely, we find a function

$$
g(j)=\frac{j(2 b-2 j+1)}{b-2 j} \cdot f(j)=\left(\begin{array}{l}
b \\
j
\end{array}\right) \frac{j(2 b-2 j+1)\left(\frac{1}{2}\right)_{j}}{d(2 b-2 d+1)\left(b-j+\frac{1}{2}\right)_{j}}
$$

with the property $g(j+1)-g(j)=f(j)$ (the latter can be easily verified). By telescoping, and by noting that $g(0)=0$, we obtain the value of the right-hand side of 6.3 :

$$
g(d)=\left(\begin{array}{l}
b \\
d
\end{array}\right) \cdot \frac{\left(\frac{1}{2}\right)_{d}}{\left(b-d+\frac{1}{2}\right)_{d}},
$$

which matches exactly the left-hand side of 6.3 .

Theorem 6.3. Let $a, b, d \in \mathbb{N}$ with $0 \leq b<a$ and $0 \leq d \leq b / 2$. Then we have

$$
c_{(a, a-b),(a-d, a-b+d)}=\frac{(2 a-b) !\left(b+\frac{1}{2}\right)\left(\frac{1}{2}\right)_{d}}{d !(a-b) !(b-d) !\left(b-d+\frac{1}{2}\right)_{a-b+d+1}} .
$$

Proof. We first show that the asserted expression is compatible with the result of Proposition 6.2 indeed, by computing the quotient

$$
\begin{aligned}
\frac{c_{(a, a-b),(a-d, a-b+d)}}{c_{(a, a-b),(a, a-b)}} & =\frac{(2 a-b) !\left(b+\frac{1}{2}\right)\left(\frac{1}{2}\right)_{d} \cdot(a-b) ! b !\left(b+\frac{1}{2}\right)_{a-b+1}}{d !(a-b) !(b-d) !\left(b-d+\frac{1}{2}\right)_{a-b+d+1} \cdot(2 a-b) !\left(b+\frac{1}{2}\right)} \\
& =\frac{b !\left(\frac{1}{2}\right)_{d}\left(b+\frac{1}{2}\right)_{a-b+1}}{d !(b-d) !\left(b-d+\frac{1}{2}\right)_{a-b+d+1}}=\left(\begin{array}{l}
b \\
d
\end{array}\right) \frac{\left(\frac{1}{2}\right)_{d}}{\left(b-d+\frac{1}{2}\right)_{d}},
\end{aligned}
$$

we see that this is the case. It remains to prove that the asserted expression is correct in the case $d=0$, i.e., when $\kappa=\lambda$. For this purpose, we employ the recursion 3.5, specialized to partitions 
with two parts:

$$
\sum_{d=0}^{a-b} c_{(a+d, a-b-d),(a, a-b)}=\left(\begin{array}{c}
2 a-b \\
a
\end{array}\right) .
$$

By dividing both sides with the binomial coefficient of the right-hand side, and by inserting the asserted closed form (after the change of variables $a \rightarrow a+d$ and $b \rightarrow b+2 d$ ), we are left with the summation identity

$$
\sum_{d=0}^{a-b} \frac{a !(a-b) !\left(b+2 d+\frac{1}{2}\right)\left(\frac{1}{2}\right)_{d}}{d !(a-b-d) !(b+d) !\left(b+d+\frac{1}{2}\right)_{a-b+1}}=1 .
$$

Taking into account the recursive definition of the coefficients $c_{\kappa, \lambda}$, the (inductive) proof is completed by verifying 6.5 . For this purpose, we denote by $f(a, b, d)$ the expression inside the sum 6.5 and construct two $\mathrm{WZ}$ pairs, i.e., two functions

$$
\begin{aligned}
g_{1}(d) & =\frac{-2 d(b+d)}{(a-b-d+1)(2 b+4 d+1)} \cdot f(a, b, d) \\
& =-\frac{a !(a-b) !\left(\frac{1}{2}\right)_{d}}{(d-1) !(b+d-1) !(a-b-d+1) !\left(b+d+\frac{1}{2}\right)_{a-b+1}}, \\
g_{2}(d) & =\frac{d(2 a+2 d+1)}{(a-b)(2 b+4 d+1)} \cdot f(a, b, d) \\
& =\frac{a !(a-b-1) !\left(\frac{1}{2}\right)_{d}}{(d-1) !(b+d) !(a-b-d) !\left(b+d+\frac{1}{2}\right)_{a-b}},
\end{aligned}
$$

such that the following identities hold (they can be verified by routine calculations):

$$
\begin{aligned}
& f(a+1, b, d)-f(a, b, d)=g_{1}(d+1)-g_{1}(d), \\
& f(a, b+1, d)-f(a, b, d)=g_{2}(d+1)-g_{2}(d) .
\end{aligned}
$$

Now we sum 6.6 for $d=0, \ldots, a-b$ and obtain

$$
\sum_{d=0}^{a-b}(f(a+1, b, d)-f(a, b, d))=g_{1}(a-b+1)-g_{1}(a, 0),
$$

or equivalently,

$$
\sum_{d=0}^{a-b+1} f(a+1, b, d)-\sum_{d=0}^{a-b} f(a, b, d)=g_{1}(a-b+1)-g_{1}(a, 0)+f(a+1, b, a-b+1) .
$$

A straightforward calculation shows that the left-hand side equals 0 , thereby showing that the sum $\sum_{d=0}^{a-b} f(a, b, d)$ is independent of $a$. Summing over 6.7), followed by a similar calculation, shows that the sum does not depend on $b$ either. Therefore, the sum in 6.5 is constant, and by setting $a=b=0$, one immediately sees that this constant is 1 .

Remark 6.4. By setting $b=a$ in Theorem 6.3 and by interpreting $(a, 0)$ as the partition $(a)$, we recover Theorem 5.1 .

$$
c_{(a),(a-d, d)}=\frac{(a) !\left(a+\frac{1}{2}\right)\left(\frac{1}{2}\right)_{d}}{d !(a-d) !\left(a-d+\frac{1}{2}\right)_{d+1}}=\left(\begin{array}{l}
a \\
d
\end{array}\right) \frac{\left(\frac{1}{2}\right)_{d}}{\left(a-d+\frac{1}{2}\right)_{d}} .
$$




\section{Partitions with three and four parts}

We have seen that the coefficients of the zonal polynomial $\mathcal{C}_{\kappa}(Y)$ are given by the row indexed by $\kappa$ in the $c_{\kappa, \lambda}$-matrix. Using (3.4) we can express all coefficients $c_{\kappa, \lambda}$ in the $\kappa$-th row as constant multiples of the diagonal coefficient $c_{\kappa, \kappa}$. Unfortunately, the latter one is harder to obtain: to apply (3.5) we need to know all $c_{\kappa, \lambda}$ in the $\lambda$-th column, which in turn are obtained by (3.4) and so on. Hence, in the worst case, we need to compute the whole triangle above the position $(\kappa, \kappa)$.

Therefore, it would be highly desirable to have a more direct way to compute the diagonal coefficients $c_{\kappa, \kappa}$. We present formulas for the special cases that $\kappa$ has three resp. four parts.

Conjecture 7.1. Let $\kappa=(a, a-b, a-c)$ with integers $0 \leq b \leq c \leq a$ be a partition of $n=3 a-b-c$ into at most three parts. Then the diagonal coefficient

$$
c_{\kappa, \kappa}=\frac{(c+1) !}{(a+1) !} \cdot \frac{n !}{\delta_{1} ! \delta_{2} ! \delta_{3} !\left(\delta_{1}+\frac{3}{2}\right)_{\delta_{2}}\left(\delta_{2}+\frac{3}{2}\right)_{\delta_{3}}},
$$

with $\delta_{1}=\kappa_{1}-\kappa_{2}=b, \delta_{2}=\kappa_{2}-\kappa_{3}=c-b$, and $\delta_{3}=\kappa_{3}-\kappa_{4}=a-c$ being the differences between consecutive parts of $\kappa$ (with the convention $\kappa_{4}=0$ ).

Conjecture 7.2. Let $\kappa=(a, a-b, a-c, a-d)$ with integers $0 \leq b \leq c \leq d \leq a$ be a partition of $n=4 a-b-c-d$ into at most four parts. Then the diagonal coefficient $c_{\kappa, \kappa}$ is given by

$$
\frac{(c+1) !(d-b+1) !}{(a-b+1) !(d+1) !\left(d+\frac{5}{2}\right)_{a-d}} \cdot \frac{n !}{\delta_{1} ! \delta_{2} ! \delta_{3} ! \delta_{4} !\left(\delta_{1}+\frac{3}{2}\right)_{\delta_{2}}\left(\delta_{2}+\frac{3}{2}\right)_{\delta_{3}}\left(\delta_{3}+\frac{3}{2}\right)_{\delta_{4}}},
$$

with $\delta_{1}=\kappa_{1}-\kappa_{2}=b, \delta_{2}=\kappa_{2}-\kappa_{3}=c-b, \delta_{3}=\kappa_{3}-\kappa_{4}=d-c$, and $\delta_{4}=\kappa_{4}-\kappa_{5}=a-d$ being the differences between consecutive parts of $\kappa$.

We have verified Conjecture 7.1 for all $0 \leq b \leq c \leq a \leq 14$ and Conjecture 7.2 for all $0 \leq b \leq c \leq d \leq a \leq 10$.

Remark 7.3. Conjecture 7.1 with $c=a$ reduces to Theorem 6.3 with $d=0$. Similarly, by letting $d=a$ in Conjecture 7.2 , we have Conjecture 7.1

\section{SageMath package for the calculation of zonal polynomials}

We briefly present the main functionality of the package Zonal. sage, which is freely available at https://jiulin90.github.io/package.html, and give a few examples. On the same website, we provide a manual with further examples of its usage.

Let $\kappa, \lambda \in \mathcal{P}_{n}$ be partitions and $Y=(a, b, c, \ldots)$ be the variables.

$\operatorname{CZonal}(\lambda, Y)$ computes the zonal polynomial $\mathcal{C}_{\lambda}(Y)$, by $(3.3)$.

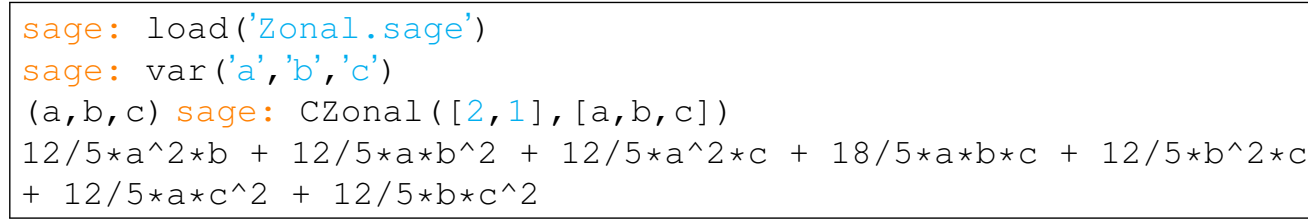

$\operatorname{MZonal}(\lambda, Y)$ computes the monomial symmetric function $M_{\lambda}(Y)$ by (3.2).

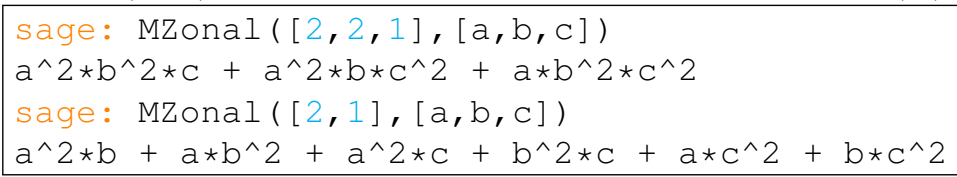


$\operatorname{Coeffi}(\kappa, \lambda)$ computes the coefficient $c_{\kappa, \lambda}$ for partitions $\kappa \geq \lambda$, by (3.4) and 3.5 .

sage: Coeffi $([5,4],[3,3,3])$

$82944 / 1925$

Remark 8.1. The SageMath software has built-in functions for Jack symmetric functions, as mentioned in the Introduction, where the zonal polynomials $\mathcal{Z}_{\lambda}(Y):=\mathcal{C}_{\lambda}(Y) / c_{\lambda, \lambda}$ are also implemented, as a special case of Jack polynomials. Many properties can be checked, such as algebraic relations among Jack polynomials in the $P, J$, and $Q$ bases. In particular, an example shows that

$$
\left(\mathcal{Z}_{(2)}(Y)\right)^{2}=\frac{64}{45} \mathcal{Z}_{(2,2)}(Y)+\frac{16}{21} \mathcal{Z}_{(3,1)}(Y)+\mathcal{Z}_{(4)}(Y)
$$

In addition, one can expand $\mathcal{Z}_{\lambda}(Y)$ into an explicit expression, by the command expand. Meanwhile, since our package only focuses on calculations of zonal polynomials, it is notably faster by using our CZonal. The screenshot below shows two computations of $\mathcal{Z}_{(4,1,1)}(a, b, c)$ and $\mathcal{C}_{(4,1,1)}(a, b, c)$, which also confirm Conjecture 7.1 with $c_{(4,1,1)(4,1,1)}=\mathcal{C}_{(4,1,1)}(a, b, c) / \mathcal{Z}_{(4,1,1)}(a, b, c)=16$.

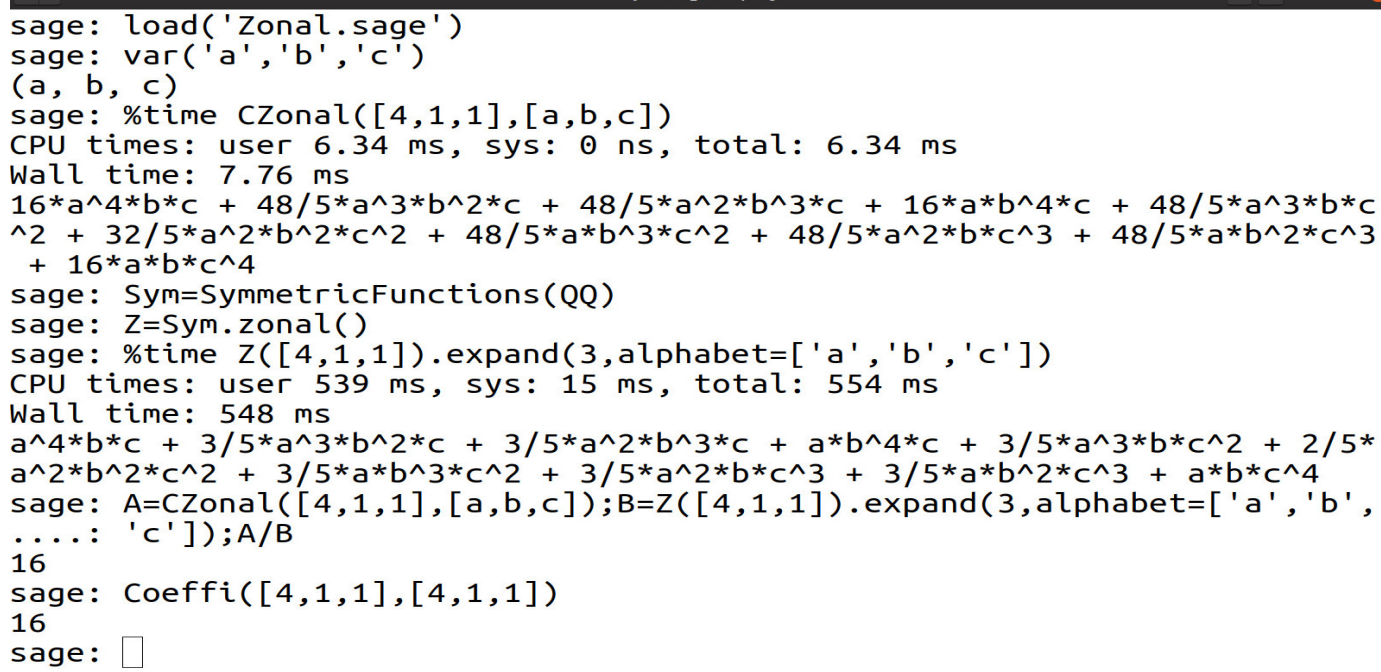

\section{Mathematica package for the calculation of zonal polynomials}

We briefly describe our implementation of zonal polynomials in Mathematica. Our software package ZonalPolynomials.m is freely available on the website www.koutschan.de/data/ zonal/. There we also provide a demo notebook with further examples of its usage. The main functions of the package are the following:

ZonalPolynomial $[\lambda]$ gives the zonal polynomial indexed by the partition $\lambda$, in terms of the monomial symmetric functions $M_{\lambda}$.

$\ln [2]:=$ ZonalPolynomial $[\{\mathbf{3}, \mathbf{2}\}]$

Out[2] $=\frac{48}{7} \mathrm{M}[3,2]+\frac{176}{21} \mathrm{M}[2,2,1]+\frac{32}{7} \mathrm{M}[3,1,1]+\frac{64}{7} \mathrm{M}[2,1,1,1]+\frac{80}{7} \mathrm{M}[1,1,1,1,1]$

ZonalPolynomial $\left[\lambda,\left\{y_{1}, \ldots, y_{m}\right\}\right]$ gives the zonal polynomial indexed by the partition $\lambda$, as a symmetric polynomial in the variables $y_{1}, \ldots, y_{m}$.

$\ln [3]:=$ ZonalPolynomial $[\{2,1\},\{a, b, c\}]$

Out[3] $=\frac{12}{5}\left(a^{2} b+a^{2} c+a b^{2}+a c^{2}+b^{2} c+b c^{2}\right)+\frac{18}{5} a b c$ 
ZonalCoefficient $[\kappa, \lambda]$ computes the zonal polynomial coefficient $c_{\kappa, \lambda}$ recursively by using 3.4 and 3.5 .

$\ln [4]==$ ZonalCoefficient $[\{8,6,6,3\},\{7,7,5,3,1\}] / /$ Timing

Out[4] $=\left\{4.60311, \frac{33426505728}{5}\right\}$

ZonalCoefficientTable $[n]$ generates a table with all zonal polynomial coefficients $c_{\kappa, \lambda}$, where $\kappa$ and $\lambda$ are partitions of $n$.

$\ln [5]:=$ ZonalCoefficientTable[4]

Out[5]= $\left\{\left\{1, \frac{4}{7}, \frac{18}{35}, \frac{12}{35}, \frac{8}{35}\right\},\left\{0, \frac{24}{7}, \frac{16}{7}, \frac{88}{21}, \frac{32}{7}\right\},\left\{0,0, \frac{16}{5}, \frac{32}{15}, \frac{16}{5}\right\},\left\{0,0,0, \frac{16}{3}, \frac{64}{5}\right\}\right.$,

$\left.\left\{0,0,0,0, \frac{16}{5}\right\}\right\}$

ZonalCoefficient $\mathbf{N}[\kappa, \lambda]$ gives a symbolic expression (a rational function in $n$ ) for the zonal coefficient $c_{\kappa, \lambda}$ in the upper left corner. The partitions $\kappa$ and $\lambda$ must be of the form $(n-i, \pi)$, where $\pi$ is a partition of $i$ and where $n$ is symbolic.

$\ln [6]:=$ ZonalCoefficientN $[\{n-3,2,1\},\{n-4,2,2\}]$

Out[6]= $\frac{4(n-3)(n-1) n\left(2 n^{2}-18 n+39\right)}{5(2 n-11)(2 n-7)}$

Acknowledgment. We are grateful to Akimichi Takemura for helpful comments, to Yi Zhang for stimulating discussions, and to Tom Koornwinder for his comments on the relation between Macdonald polynomials and Jack polynomials. The first author also would like to thank Raymond Kan, who, after the submission of the original draft, contributed to the SageMath program and now becomes a co-contributor. Both authors were supported by the Austrian Science Fund (FWF): P29467-N32, and the second author was also supported by F5011-N15.

\section{References}

[1] Nist digital library of mathematical functions. http://dlmf.nist.gov/, Release 1.0.20 of 2018-09-15. F. W. J. Olver, A. B. Olde Daalhuis, D. W. Lozier, B. I. Schneider, R. F. Boisvert, C. W. Clark, B. R. Miller and B. V. Saunders, eds.

[2] Ronald Butler and Robert Paige. Exact distributional computations for Roy's statistic and the largest eigenvalue of a Wishart distribution. Statistics and Computing, 21:147-157, 2011.

[3] Kenneth Gross and Donald Richards. Special functions of matrix argument. I. Algebraic induction, zonal polynomials, and hypergeometric functions. Transactions of the AMS, 301(2):781-811, 1987.

[4] Hiroki Hashiguchi, Yasuhide Numata, Nobuki Takayama, and Akimichi Takemura. The holonomic gradient method for the distribution function of the largest root of a Wishart matrix. Journal of Multivariate Analysis, 117:296-312, 2013.

[5] Sigurdur Helgason. Differential Geometry and Symmetric Spaces. Academic Press, 1962.

[6] R. Gutiérrez Jáimez and J. A. Mermoso Gutiérrez. An application of zonal polynomials to the generalization of probability distributions. Linear Algebra and its Applications, 121:610-616, 1989.

[7] A. James. Calculation of zonal polynomial coefficients by use of the Laplace-Beltrami operator. The Annals of Mathematical Statistics, 39(5):1711-1718, 1968.

[8] Iain Johnstone. On the distribution of the largest eigenvalue in principal components analysis. The Annals of Statistics, 29(2):295-327, 2001.

[9] Manuel Kauers. Guessing handbook. Technical Report 09-07, RISC Report Series, Johannes Kepler University, Linz, Austria, 2009. http://www.risc.jku.at/research/combinat/software/Guess/.

[10] Plamen Koev and Alan Edelman. The efficient evaluation of the hypergeometric function of a matrix argument. Mathematics of Computation, 75(254):833-846, 2006. 
[11] Christoph Koutschan. HolonomicFunctions (user's guide). Technical Report 10-01, RISC Report Series, Johannes Kepler University, Linz, Austria, 2010. http://www.risc.jku.at/research/combinat/software/ HolonomicFunctions/.

[12] H. B. Kushner and Morris Meisner. Formulas for zonal polynomials. Journal of Multivariate Analysis, 14:336-347, 1984.

[13] Maher Moakher. A differential geometric approach to the geometric mean of symmetric positive-definite matrices. SIAM Journal on Matrix Analysis and Applications, 26(3):735-747, 2005.

[14] Robb Muirhead. Systems of partial differential equations for hypergeometric functions of matrix argument. The Annals of Mathematical Statistics, 41(3):991-1001, 1970.

[15] Robb Muirhead. Aspects of multivariate statistical theory. Wiley series in probability and mathematical statistics. Probability and mathematical statistics. John Wiley \& Sons, New York, 1982.

[16] Hiromasa Nakayama, Kenta Nishiyama, Masayuki Noro, Katsuyoshi Ohara, Tomonari Sei, Nobuki Takayama, and Akimichi Takemura. Holonomic gradient descent and its application to the Fisher-Bingham integral. Advances in Applied Mathematics, 47(3):639-658, 2011.

[17] Masayuki Noro. System of partial differential equations for the hypergeometric function 1F1 of a matrix argument on diagonal regions. In Proceedings of the International Symposium on Symbolic and Algebraic Computation (ISSAC), ISSAC' 16, pages 381-388, New York, NY, USA, 2016. ACM.

[18] Constantin Siriteanu, Akimichi Takemura, Satoshi Kuriki, Hyundong Shin, and Christoph Koutschan. MIMO zero-forcing performance evaluation using the holonomic gradient method. IEEE Transactions on Wireless Communications, 14(4):2322-2335, 2015.

[19] John Stembridge. A Maple package for symmetric functions. Journal of Symbolic Computation, 20:755$768,1995$.

[20] Akimichi Takemura. Zonal Polynomials, volume 4 of Institute of Mathematical Statistics Lecture Notes Monograph Series. Institute of Mathematical Statistics, Hayward, CA, 1984.

[21] John Wishart. The generalised product moment distribution in samples from a normal multivariate population. Biometrika, 20A(1-2):32-52, 1928.

Lin Jiu

Department of Mathematics and Statistics,Dalhousie University, 6316 Coburg Road, Halifax, Nova Scotia, Canada B3H 4R2

Lin.Jiu@dal.ca

Christoph Koutschan

Johann Radon Institute for Computational and Applied Mathematics (RICAM), Altenberger Straße 69, A-4040

Linz, Austria

christoph.koutschan@ricam.oeaw.ac.at 